\title{
Reliability Optimization of Electrical Power Systems Subject to Severe Environmental Loads
}

\author{
Chunyang $\mathrm{Li}^{\mathrm{a}^{*}}$, Shouhua Chen, Lingwei $\mathrm{Li}$ \\ Academy of Armored Force Engineering, Beijing 100072, China \\ ab998003@163.com
}

Keywords: multi-state systems; environmental loads; optimization; universal generating function; genetic algorithms

\begin{abstract}
Electrical power supply interruption caused by severe environmental loads (such as ice storms, snowstorms, cyclones and earthquakes) resulted in great economic losses. The optimal problem of electrical power systems subject to this kind of common cause failures is studied to provide the desired level of reliability with minimum cost. The system reliability is estimated by the universal generating function method, and the optimal model is solved by a genetic algorithm. A numerical example is illustrated to demonstrate the proposed method.
\end{abstract}

\section{Introduction}

Electrical power systems play an important role in our society, and the reliability of electrical power systems is very important. Faced with severe environmental loads, such as ice storms, snowstorms, earthquakes and cyclones, greater challenges are imposed on the reliability and safety of electrical power systems, and the electricity supply may be cut off, which will result in great economic losses. We can strengthen electrical power systems, but this will cost a lot of money that we cannot afford. Then methods of optimizing electrical power systems structures must be taken to improve their ability against disasters with the limited budget.

The failure of lots of transmission towers caused by severe environmental loads is a kind of common cause failures (CCFs). CCFs are the simultaneous failure of multiple components due to a common cause (CC). The origin of $\mathrm{CC}$ events includes environmental loads, errors in maintenance and system design flaws. Researchers proposed methods to analyze the reliability of binary systems subject to CCFs [1-3]. But electrical power systems are multi-state systems (MSSs) which can work in different performance levels from perfect functioning to complete failure $[4,5]$. So the optimal problem of the electrical power system is how to analyze the system reliability and optimize the system structure when the MSS consists of multi-state components subject to CCFs.

Gregory Levitin $[6,7]$ have researched the reliability analysis of MSSs subject to CCFs, but the subsystems of MSSs in [6,7] consist of binary components. Extensive research on reliability optimization of MSSs has been done [8-10]. Besides, Y. Massim et al. [11] and Rabah Ouiddir et al. [12] researched the optimal problem of multi-state electrical power systems. But the components of MSSs in these papers fail mutually independently, and CCFs are not considered. Up to now, there are few papers including reliability optimization of MSSs subject to CCFs.

In this paper, the reliability of MSSs subject to CCFs caused by severe environmental loads is analyzed, and the optimal problem is solved.

\section{Problem Formulation}

General assumptions:

(1) The components are non-repairable.

(2) All components will fail when severe environmental loads are beyond the design limit of components.

General Description of the Problem. A multi-state system containing $N$ subsystems connected in series is depicted in Figure 1. There are ${ }^{h_{i}}$ different types of components connected in parallel 
which can be selected from $H_{i}$ different types of components in subsystem ${ }^{i}$. There are $n_{i j}$ components of type $j$ and totally $n_{i}$ components used in subsystem $i, j=k_{1}, k_{2}, \cdots, k_{h_{i}}, \sum_{j=k_{1}}^{k_{k_{h}}} n_{i j}=n_{i}$. The performance of components is characterized by capacity $\mathbf{g}_{i j}$, availability $\mathbf{q}_{i j}$ and cost $c_{i j}$. The cost of the system is $C$, and the availability of the system is $A_{s}$.

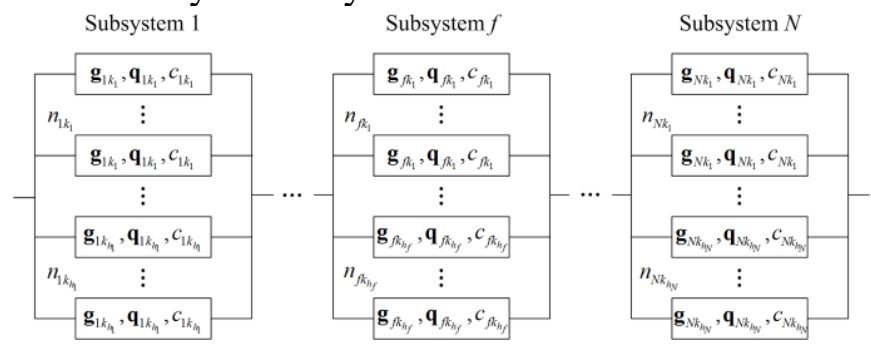

Figure 1. Structure of a multi-state series-parallel system.

To analyze the effect of CCFs on the system reliability, assume that subsystem $f$ is subject to severe environmental loads. When the environmental loads are beyond the design limit of components of the subsystem $f$, all components of this type will fail.

The Measure of Electrical Power System Reliability. In electrical power systems, the loss of load probability (LOLP) index is a commonly used measure for reliability estimation. LOLP is defined as the probability that the system cannot supply a given demand load [11]. Suppose that the operation period is $T$ which is divided into $D$ intervals. Each interval has a duration $T_{d}$ and a required demand level $W_{d}, d=1,2, \cdots D$. Then the LOLP can be defined as

$$
\operatorname{LOLP}=\sum_{d=1}^{D} P\left(G_{s}<W_{d}\right) T_{d} / T
$$

Where $P\left(G_{s}<W_{d}\right)$ is the probability that the system capacity $G_{s}$ is lower than the demand level $W_{d}$. To obtain a measure of the electrical power system reliability, the reliability index $E_{s}$ is introduced

$$
E_{s}=1-\mathrm{LOLP}=\sum_{d=1}^{D} P\left(G_{s} \geq W_{d}\right) T_{d} / T
$$

Mathematics Model. The objective is to minimize the cost of the MSS subject to the specified minimum required reliability index $E_{0}$. The optimal model of the system can be formulated as:

$$
\begin{array}{cc}
\text { Min } C=\sum_{i=1}^{N} \sum_{j=k_{1}}^{k_{k_{i j}}} n_{i j} c_{i j} \\
\text { s.t. } \quad E_{s} \geq E_{0} \\
& n_{i j} \in Z^{+}
\end{array}
$$

Where $j$ is the type of components used in subsystem $i ; n_{i j}$ is the number of components type $j$ used in subsystem $i ; k_{1}, k_{2}, \cdots, k_{h_{i}}$ is the version number of components used; $Z^{+}$is the space discrete of positive integers. $i=1,2, \cdots, N$.

\section{The Reliability Estimation of the System}

The Availability of Subsystems without CCFs. The UGF method is widely used in the MSS availability estimation, and it is proven to be very effective for high-dimension combinatorial problems. Based on the UGF method, the MSS availability can be obtained as a function of system structure, performance and availability characteristics of its components $[4,5]$.

The UGF of components type $j$ in subsystem $i$ is defined as a polynomial

$$
U_{i j}(z)=\sum_{l=1}^{M} q_{i j l} z^{g_{j i l}}
$$


Where $M$ is the number of possible states; $\mathbf{g}_{i j}=\left\{g_{i j 1}, g_{i j 2}, \cdots, g_{i j M}\right\}$ is the capacity, and the corresponding probability is $\mathbf{q}_{i j}=\left\{q_{i j 1}, q_{i j 2}, \cdots, q_{i j M}\right\} ; q_{i j l}=\operatorname{Pr}\left\{G_{i j}=g_{i j l}\right\}, G_{i j}$ is the capacity of the component.

Define the $\sigma$ operator as

$$
\sigma\left(U_{1}(z), U_{2}(z)\right)=\sum_{l=1}^{M} \sum_{m=1}^{M} q_{1 l} \cdot q_{2 m} \cdot z^{g_{11}+g_{2 m}}
$$

Then the UGF of the subsystem $i$ without CCF can be computed using the $\sigma$ operator consecutively:

$$
U_{i}(z)=\sigma\left(U_{i 1}(z), \cdots, U_{i i_{i}}(z)\right)=\sum_{s=1}^{M_{i}} q_{i s} z^{G_{i s}}
$$

Where $M_{i}$ is the number of possible states of the subsystem $i ; G_{i s}$ is the capacity of the subsystem $i ; q_{i s}$ is the corresponding probability.

Define the following $\delta$ operator over $U_{i}(z)$ :

$$
\begin{gathered}
\delta\left(U_{i}(z), W_{d}\right)=\delta\left(\sum_{s=1}^{M_{i s}} q_{i s} z^{G_{i s}}, W_{d}\right)=\sum_{s=1}^{M_{i s}} \delta\left(q_{i s} z^{G_{i s}}, W_{d}\right) \\
\delta\left(q_{i s} z^{G_{i s}}, W_{d}\right)= \begin{cases}q_{i s}, & G_{i s} \geq W_{d} \\
0, & G_{i s}<W_{d}\end{cases}
\end{gathered}
$$

Then the availability of the subsystem $i$ satisfying a given demand level $W_{d}$ is given by

$$
A_{i}\left(W_{d}\right)=\operatorname{Pr}\left\{G_{i s} \geq W_{d}\right\}=\delta\left(U_{i}(z), W_{d}\right)=\sum_{G_{i s} \geq W_{d}} q_{i s}
$$

The Availability of Subsystems with CCFs. When the environmental loads are beyond the design limit of components, all components of this type will fail. Incorporating CCFs into the MSSs, the procedure of reliability estimation should be improved.

In the subsystem $f$, there are $h_{f}$ different types of components used whose limit loads are $l_{k_{1}}, \cdots, l_{k_{h_{f}}}\left(l_{k_{1}}<\cdots<l_{k_{h_{f}}}\right)$, respectively. When the subsystem $f$ is subject to CCFs, the availability of the subsystem $f$ satisfying a specified demand level $W_{d}$ is given by

$$
\begin{aligned}
A_{f}\left(W_{d}\right) & =\tilde{A}_{f}\left(W_{d}\right) \times\left(1-C C_{k_{1}}-C C_{k_{1}, k_{2}}-\cdots-C C_{k_{1}, \cdots, k_{h_{f}}}\right)+\tilde{A}_{f k_{1}}\left(W_{d}\right) \times C C_{k_{1}}+\tilde{A}_{f k_{1}, f k_{2}}\left(W_{d}\right) \times C C_{k_{1}, k_{2}}+\cdots \\
& +\tilde{A}_{f k_{1}, \cdots, f k_{k_{f}}}\left(W_{d}\right) \times C C_{k_{1}, \cdots, k_{h_{f}}}
\end{aligned}
$$

Where $\tilde{A}_{f}\left(W_{d}\right)$ is the availability of the subsystem $f$ without CCFs; $k_{1}, k_{2}, \cdots, k_{h_{f}}$ is the type of components used in the subsystem $f ; C C_{k_{1}}$ is the probability that all components type $k_{1}$ fail due to a CC; $C C_{k_{1}, k_{2}}$ is the probability that all components type $k_{1}$ and $k_{2}$ fail due to a CC; $C C_{k_{1}, \cdots, k_{h f}}$ is the probability that all components (type $k_{1}, k_{2}, \cdots, k_{h_{f}}$ ) fail due to a CC; $\tilde{A}_{f k_{1}}\left(W_{d}\right)$ is the availability of the subsystem $f$ when all components type $k_{1}$ fail due to a CC; $\tilde{A}_{f k_{1}, f k_{2}}$ is the availability of the subsystem $f$ when all components type $k_{1}$ and $k_{2}$ fail due to a CC; $\tilde{A}_{f k_{1}, \cdots, f k_{k_{f}}}\left(W_{d}\right)$ is the availability of the subsystem $f$ when all components fail due to a CC. Obviously, $\tilde{A}_{f k_{1}, \cdots, f k_{h_{f}}}\left(W_{d}\right)=0$.

The Reliability Index of the Electrical Power System. The system is a series-parallel system and consists of subsystems in series. The availability of all the subsystems in the system can be obtained using the UGF method, so the availability of the system is

$$
A_{s}\left(W_{d}\right)=P\left(G_{s} \geq W_{d}\right)=\prod_{i=1}^{N} A_{i}\left(W_{d}\right)
$$

According to Eq.(2), the reliability index of the electrical power system is

$$
E_{s}=\sum_{d=1}^{D} P\left(G_{s} \geq W_{d}\right) T_{d} / T=\sum_{d=1}^{D} A_{s}\left(W_{d}\right) T_{d} / T
$$

Where $G_{s}$ is the capacity of the system. 


\section{GENETIC ALGORITHMS}

The optimal model can be solved by GA. The representation of chromosomes is that of integer strings, just like this: $\mathbf{v}_{k}=\left(r_{11}, r_{12}, \cdots, r_{1 H_{1}}, r_{21}, r_{22}, \cdots, r_{2 H_{2}}, \cdots, r_{N 1}, r_{N 2}, \cdots, r_{N H_{N}}\right)$.The $r_{i j}$ denotes the redundancy number of components type $j$ in subsystem $i$.

To provide an efficient search through the infeasible region but to assure that the final best solution is feasible, the fitness function is defined as the sum of the objective and a penalty function.

\section{Illustrative Example}

The following example illustrates the application of the method suggested for solving the problem of electrical power systems. The electrical power system was introduced by Y. Massim et al. [11]. The system is composed of five subsystems connected in series. The electrical power is generated from the station units (subsystem 1) which are multi-state. Then transformed for high voltage (HT) by the HT transformers (subsystem 2) and carried by the HT lines (subsystem 3). A second transformation occurs in HT/MT transformers (subsystem 4), which supply the MT load by the MT lines (subsystem 5).

In this electrical power system, transmission towers are not considered explicitly. To analyze the effect of CCFs caused by severe environmental loads on the system reliability, transmission towers which are used in HT lines (subsystem 3) and MT lines (subsystem 5) are included in this study. So the subsystems 3 and 5 may be suffered from CCFs caused by ice storms. The characteristics of the products available on the market for each type of device are presented in Table 1.

\begin{tabular}{|c|c|c|c|c|}
\hline Subsystem & Components & Capacity (\%) & Availability & Cost (Million \$) \\
\hline \multirow{8}{*}{ Power units } & \multirow{2}{*}{$\mathrm{T} 1$} & 48 & 0.820 & \multirow{2}{*}{8.202} \\
\hline & & 35 & 0.172 & \\
\hline & \multirow{2}{*}{$\mathrm{T} 2$} & 30 & 0.730 & \multirow{2}{*}{6.895} \\
\hline & & 22 & 0.260 & \\
\hline & \multirow{2}{*}{$\mathrm{T} 3$} & 28 & 0.874 & \multirow{2}{*}{6.354} \\
\hline & & 20 & 0.123 & \\
\hline & \multirow{2}{*}{$\mathrm{T} 4$} & 24 & 0.660 & \multirow{2}{*}{5.965} \\
\hline & & 16 & 0.325 & \\
\hline \multirow{5}{*}{ HT transformers } & $\mathrm{T} 1$ & 45 & 0.978 & 2.555 \\
\hline & $\mathrm{T} 2$ & 38 & 0.969 & 2.215 \\
\hline & T3 & 34 & 0.985 & 2.202 \\
\hline & $\mathrm{T} 4$ & 28 & 0.942 & 1.707 \\
\hline & T5 & 22 & 0.935 & 1.203 \\
\hline \multirow{8}{*}{$\begin{array}{l}\text { HT lines and } \\
\text { towers }\end{array}$} & $\mathrm{T}^{\mathrm{a}}$ & 42 & 0.979 & 6.404 \\
\hline & $\mathrm{T} 2^{\mathrm{a}}$ & 36 & 0.993 & 6.192 \\
\hline & $\mathrm{T}^{\mathrm{a}}$ & 30 & 0.993 & 5.939 \\
\hline & $\mathrm{T}^{\mathrm{a}}$ & 25 & 0.991 & 5.622 \\
\hline & $\mathrm{T}^{\mathrm{b}}$ & 42 & 0.979 & 9.787 \\
\hline & $\mathrm{T}^{\mathrm{b}}$ & 36 & 0.993 & 9.575 \\
\hline & $\mathrm{T} 7^{\mathrm{b}}$ & 30 & 0.993 & 9.322 \\
\hline & $\mathrm{T}^{\mathrm{b}}$ & 25 & 0.991 & 9.005 \\
\hline \multirow{5}{*}{$\begin{array}{l}\text { HT/MT } \\
\text { transformers }\end{array}$} & $\mathrm{T} 1$ & 40 & 0.989 & 1.402 \\
\hline & $\mathrm{T} 2$ & 36 & 0.990 & 1.272 \\
\hline & T3 & 36 & 0.982 & 1.234 \\
\hline & $\mathrm{T} 4$ & 28 & 0.987 & 1.064 \\
\hline & $\mathrm{T} 5$ & 24 & 0.985 & 0.935 \\
\hline \multirow{8}{*}{$\begin{array}{l}\text { MT lines and } \\
\text { towers }\end{array}$} & $\mathrm{T} 1^{\mathrm{a}}$ & 35 & 0.985 & 3.770 \\
\hline & $\mathrm{T}^{\mathrm{a}}$ & 32 & 0.980 & 3.614 \\
\hline & $\mathrm{T}^{\mathrm{a}}$ & 26 & 0.982 & 3.328 \\
\hline & $\mathrm{T} 4^{\mathrm{a}}$ & 24 & 0.979 & 3.302 \\
\hline & $\mathrm{T}^{\mathrm{b}}$ & 35 & 0.985 & 5.462 \\
\hline & $\mathrm{T}^{\mathrm{b}}$ & 32 & 0.980 & 5.306 \\
\hline & $\mathrm{T} 7^{\mathrm{b}}$ & 26 & 0.982 & 4.990 \\
\hline & $\mathrm{T}^{\mathrm{b}}$ & 24 & 0.979 & 4.964 \\
\hline
\end{tabular}

${ }^{a}$ These kinds of transmission towers can withstand $20 \mathrm{~mm}$ of ice. ${ }^{\mathrm{b}}$ These kinds of transmission towers can withstand $40 \mathrm{~mm}$ of ice. 
The capacities are given as a percentage of the maximum demand level. The failure state is evidently not reported in the table. From Table 1 we know that, if we strengthen the transmission towers to avoid the CCFs caused by ice storms, we have to invest much more money.

Table 2 shows different demand levels for a given period. This is usually known for every system as the cumulative load-demand curve. The minimum demand level is $288 \mathrm{MW}$, which happens mostly in winter. When the components of the system are suffered from ice storms that are beyond the design limit, transmission towers will fail. According to statistics, the probability that 20 $\mathrm{mm}$ of ice forms on power lines is about $2 \%$ in winter, and the probability of $40 \mathrm{~mm}$ of ice forming is about $1 \%$.

Table2. Parameters of cumulative load-demand curve
\begin{tabular}{|l|l|}
\hline Load (MW) & Duration (h) \\
\hline 450 & 4380 \\
\hline 405 & 1460 \\
\hline 324 & 2200 \\
\hline 288 & 720 \\
\hline
\end{tabular}

The objective is to minimize the cost of the electrical power system, subject to the reliability index of the system no less than 0.975 when the performance of the system meets the given demand.

Two situations are studied:

(1) Strengthened transmission towers are used.

(2)None of the strengthened transmission towers (components type 5, 6, 7 and 8 in the subsystems 3 and 5) is used.

The results of reliability optimization of these two situations are listed in Table 3. The results indicate that the cost of the electrical power system is higher when strengthened transmission towers are selected. So when electrical power systems are built, the transmission towers which can withstand 20 40 $\mathrm{mm}$ of ice forming on power lines usually are not used for the purpose of cost saving. But the problem is that, when the ice storm arrives and the ice forming on power lines is above $20 \mathrm{~mm}$, a lot of transmission towers will be pulled down and great economic losses will be caused. If the direct economic losses reach 12 million dollars when the electricity supply is absolutely cut off, the optimal result of the second situation is better. Otherwise, the optimal result of the first situation is better.

Table 3. Convergence results of the two situations

\begin{tabular}{|c|c|c|c|c|c|c|c|c|c|c|c|}
\hline \multirow{2}{*}{ Situation } & \multirow{2}{*}{ Subsystem } & \multicolumn{8}{|c|}{ Component choice } & \multirow{2}{*}{$E_{S}$} & \multirow{2}{*}{$C$ (Million \$) } \\
\hline & & T1 & T2 & T3 & T4 & T5 & T6 & T7 & T8 & & \\
\hline \multirow{5}{*}{1} & 1 & 2 & 1 & 0 & 0 & & & & & \multirow{5}{*}{0.976} & \multirow{5}{*}{105.520} \\
\hline & 2 & 3 & 1 & 1 & 0 & 4 & & & & & \\
\hline & 3 & 0 & 3 & 0 & 1 & 0 & 0 & 0 & 1 & & \\
\hline & 4 & 0 & 3 & 0 & 2 & 2 & & & & & \\
\hline & 5 & 1 & 1 & 2 & 0 & 0 & 1 & 0 & 1 & & \\
\hline \multirow{5}{*}{2} & 1 & 3 & 0 & 0 & 0 & & & & & \multirow{5}{*}{0.982} & \multirow{5}{*}{94.376} \\
\hline & 2 & 0 & 1 & 0 & 5 & 1 & & & & & \\
\hline & 3 & 1 & 1 & 1 & 2 & & & & & & \\
\hline & 4 & 0 & 3 & 0 & 4 & 2 & & & & & \\
\hline & 5 & 2 & 2 & 1 & 0 & & & & & & \\
\hline
\end{tabular}

If CCFs are not considered, the reliability index of the system composed of components gained by the two situations is 0.978 and 0.985 , respectively. So CCFs reduce the system reliability. If CCFs happen more frequently, the effect of CCFs on the system reliability will be much larger.

\section{Conclusions}

The system reliability analysis and optimization of MSSs subject to CCFs has not been researched before. In this paper, a procedure is proposed to analyze the reliability and optimize the structure of the multi-state electrical power system subject to severe environmental loads. This procedure is based on the UGF method and GA, and the method is researched to analyze the reliability of subsystems with CCFs. The results of the illustrative example show that the effect of 
components redundancy is reduced and the strengthening of transmission towers is necessary when CCFs are considered. To improve the anti-disaster ability of electrical power systems with the limited budget, the structure of the electrical power systems should be optimized and towers of some transmission lines should be strengthened.

\section{References}

[1]Jussi K. Vaurio, An implicit method for incorporating common-cause failures in system analysis, IEEE Transactions on Reliability, 47 (1998) 173-180,.

[2]Liudong Xing, Reliability evaluation of phased-mission systems with imperfect fault coverage and common-cause failures, IEEE Transactions on Reliability, 56 (2007) 58-68.

[3]L. Xie, J. Zhou, and X. Wang, Data mapping and the prediction of common cause failure probability, IEEE Transactions on Reliability, 54 (2005) 291-296.

[4]Gregory Levitin, The universal generating function in reliability analysis and optimization, Springer,London, 2005.

[5]Anatoly Lisnianski and Gregory Levitin, Multi-state system reliability: Assessment, optimization and applications, World Scientific, Singapore, 2003.

[6]Gregory Levitin, Incorporating common-cause failures into nonrepairable multistate seriesparallel system analysis, IEEE Transactions on Reliability, 50 ( 2001) 380-388.

[7]Gregory Levitin, Common supply failures in linear multi-state sliding window systems, Reliability Engineering and System Safety, 82 (2003) 55-62.

[8]Zhigang Tian and Ming J. Zuo, Redundancy allocation for multi-state systems using physical programming and genetic algorithms, Reliability Engineering and System Safety, 91 (2006) 10491056.

[9]Mohamed Ouzineb, Mustapha Nourelfath, and Michel Gendreau, Tabu search for the redundancy allocation problem of homogenous series-parallel multi-state systems, Reliability Engineering and System Safety, 93 (2008) 1257-1272.

[10]Manju Agarwal and Rashika Gupta, Homogeneous redundancy optimization in multi-state series-parallel systems: A heuristic approach, IIE Transactions, 39 (2007) 277-289.

[11]Y. Massim, A. Zeblah, R. Meziane, M. Benguediab, and A. Ghouraf, Optimal design and reliability evaluation of multi-state series-parallel power systems, Nonlinear Dynamics, 40 (2005) 309-321.

[12]Rabah Ouiddir, Mostefa Rahli, Rachid Meziane, and Abdelkader Zeblah, Ant colony optimization for new redesign problem of multi-state electrical power systems, Journal of Electrical Engineering, 55 (2004) 57-63. 